\title{
A New Model for Applying Extended Kalman Filtering to Extract Harmonic Signals from Noisy Measurements
}

\author{
Ming Li, Manus Henry and Stephen Duncan \\ Department of Engineering Science \\ University of Oxford \\ Oxford OX1 3PJ \\ United Kingdom \\ Email: \{ming.li,manus.henry,stephen.duncan\}@eng.ox.ac.uk
}

\begin{abstract}
The extended Kalman filter has been used to estimate a harmonic signal from noisy measurements. Most algorithms are based on the Cartesian model, which is a discretization in time of the continuous state space model associated with the differential equation that is satisfied by a sinusoidal signal with constant amplitude and frequency. In order to handle the more realistic case where both amplitude and frequency are changing, this basic model is modified by including ad hoc extensions. This paper starts by deriving a differential equation that explicitly includes time varying amplitude and frequency, and it is shown that this can be reduced to a Bessel's equation of order $1 / 2$ that has a closed form solution. This is used to derive an explicit expression for a discrete-time model, which forms the basis of an extended Kalman filter. Simulation results show that this algorithm outperforms other approaches, particularly for harmonic signals where the frequency is changing rapidly.
\end{abstract}

\section{INTRODUCTION}

This paper considers the problem of tracking a harmonic signal of the form

$$
s(t)=\alpha(t) \cos \phi(t)
$$

where $\alpha(t)$ is the amplitude of the signal and $\phi(t)$ is its phase, which is determined by the frequency

$$
\phi(t)=\phi(0)+\int_{0}^{t} \phi\left(t_{1}\right) \mathrm{d} t_{1} .
$$

Given noisy measurements of the harmonic signal that are taken at discrete time intervals

$$
y(k T)=s(k T)+n(k T)
$$

for $k \in \mathbb{Z}^{+}$, where $T$ is the interval between samples and $n(k T) \sim \mathcal{N}(0, R)$ is zero-mean, Gaussian, white noise with variance $R \in \mathbb{R}$, the aim is to generate $\hat{s}(T)$ the estimate of the underlying signal at each of the sampling points, together with estimates of $\alpha(k T)$ and $\omega(k T)$. This problem was motivated by the need to track sinusoidal signals in Coriolis flow metering [1], but is also encountered in applications such as power systems, speech recognition and communications [2], [3], [4], [5], [6], [7], [8], [9].
When both the frequency and the amplitude of the harmonic signal $\omega(t)$ are constant, then the signal in (1) satisfies

$$
s^{\prime \prime}(t)+\omega^{2} s(t)=0
$$

where' denotes differentiation with respect to time. This can be expressed as a state space system

$$
\frac{\mathrm{d}}{\mathrm{d} t}\left[\begin{array}{c}
s(t) \\
s^{\prime}(t)
\end{array}\right]=\left[\begin{array}{cc}
0 & 1 \\
-\omega^{2} & 0
\end{array}\right]\left[\begin{array}{c}
s(t) \\
s^{\prime}(t)
\end{array}\right],
$$

which can be discretised by integrating over $t \in[k T,(k+1) T]$

$$
\left[\begin{array}{c}
s[(k+1) T] \\
s^{\prime}[(k+1) T]
\end{array}\right]=\left[\begin{array}{cc}
\cos \omega T & \frac{1}{\omega} \sin \omega T \\
-\omega \sin \omega T & \cos \omega T
\end{array}\right]\left[\begin{array}{c}
s(k T) \\
s^{\prime}(k T)
\end{array}\right] .
$$

The variation in amplitude of the signal can be represented by an additive state noise term $w(k T) \sim \mathcal{N}(0, Q)$, which is assumed to enter the system at each sample time, $t=k T$, where $w(k T)$ is zero-mean, Gaussian, white noise with covariance matrix $Q \in \mathbb{R}^{2}$. Since the state noise only enters at the sampling times, then the amplitude of the signal will be constant between samples and for constant $\omega$, then

$$
s^{\prime}(t)=\omega \alpha(k T) \sin \omega t
$$

for $t \in[k T,(k+1) T]$. It is convenient to define an alternative state $x(t) \in \mathbb{R}^{2}$

$$
\left[\begin{array}{l}
x_{1}(k T) \\
x_{2}(k T)
\end{array}\right]=\left[\begin{array}{c}
\alpha(k T) \cos \omega k T \\
\alpha(k T) \sin \omega k T
\end{array}\right],
$$

which can be obtained by transforming the state in (6)

$$
\left[\begin{array}{l}
x_{1}(k T) \\
x_{2}(k T)
\end{array}\right]=\left[\begin{array}{cc}
1 & 0 \\
0 & -\frac{1}{\omega}
\end{array}\right]\left[\begin{array}{c}
s(k T) \\
s^{\prime}(k T)
\end{array}\right]
$$

so that

$$
\begin{aligned}
{\left[\begin{array}{l}
x_{1}[(k+1) T] \\
x_{2}[(k+1) T]
\end{array}\right]=} & {\left[\begin{array}{cc}
\cos \omega T & -\sin \omega T \\
\sin \omega T & \cos \omega T
\end{array}\right]\left[\begin{array}{c}
x_{1}(k T) \\
x_{2}(k T)
\end{array}\right] } \\
& +\left[\begin{array}{c}
w_{1}(k T) \\
w_{2}(k T)
\end{array}\right]
\end{aligned}
$$


This is referred to as the Cartesian model. The model is completed by applying the state transformation to the measurement equation (3)

$$
y(k T)=\left[\begin{array}{ll}
1 & 0
\end{array}\right]\left[\begin{array}{l}
x_{1}(k T) \\
x_{2}(k T)
\end{array}\right]+n(k T)
$$

and when $\omega$ is known, a Kalman filter can be used to estimate $x_{1}(k T)$ and $x_{2}(k T)$, which can be combined using (8) to provide an estimate of $\alpha(k T)$.

The more challenging problem, which is addressed in this paper, is to estimate the signal when the frequency is both unknown and changing. The simplest approach is to modify the Cartesian model in (10) by extending the state to include the frequency, so that

$$
\begin{aligned}
& {\left[\begin{array}{l}
x_{1}[(k+1) T] \\
x_{2}[(k+1) T] \\
x_{3}[(k+1) T]
\end{array}\right]=} \\
& \quad\left[\begin{array}{ccc}
\cos x_{3}(k T) T & -\sin x_{3}(k T) T & 0 \\
\sin x_{3}(k T) T & \cos x_{3}(k T) T & 0 \\
0 & 0 & 1
\end{array}\right]\left[\begin{array}{l}
x_{1}(k T) \\
x_{2}(k T) \\
x_{3}(k T)
\end{array}\right] \\
& +\left[\begin{array}{c}
w_{1}(k T) \\
w_{2}(k T) \\
w_{3}(k T)
\end{array}\right],
\end{aligned}
$$

where $x_{3}(k T)=\omega(k T)$. Because this model is non-linear, estimates of $x_{1}(k T), x_{2}(k T)$ and $\omega(k T)$ can be obtained using an extended Kalman filter (EKF) and this approach has been studied extensively [13]. The model also forms the basis of a related approach based on adaptive notch filtering [18], [19], [20], [21]. Although a stability analysis of an EKF that is based on a modified form of this model has been developed in [22], it is only possible to show that the EKF is locally asymptotically stable and the stability is dependent on the frequency of the signal not changing "too rapidly".

In an attempt to overcome this limitation, [15] introduced the modified Cartesian model (MCM), which extended the state vector in the model to include $\omega^{\prime}(k T)$, so that $\omega(t)$ is allowed to vary (linearly) over $t \in[k T,(k+1) T]$. This does improve the ability of the extended Kalman filter to track changing frequencies, but is still not able to follow very rapid changes in frequency. One possible reason for this is that EKF's which use either the augmented Cartesian model or the modified Cartesian model, are extensions of the model in (10), which in turn, is a discretization of the continuous state space model in (4) that is derived from the differential equation in (5). However, this differential equation is only applicable when the frequency and the amplitude of the harmonic signal is constant, which is not the situation that we are trying to model.

One approach that avoids this limitation was introduced in $\mathrm{Ng}$ et al. [16], who used concepts from target tracking. In their model, which is referred to as the nearly constant frequency (NCF) model, the state consists of three terms,

$$
x(k T)=[\phi(k T) \omega(k T) \alpha(k T)]
$$

The update equation for these states is linear, but the nonlinearity is introduced in the measurement equation, which takes the form

$$
y(k T)=x_{3}(k T) \cos x_{1}(k T)
$$

They also consider the case where the state vector is extended to include $\omega^{\prime}(k T)$ to allow for $\omega(t)$ varying between samples.

The approach taken in this paper is to return to the original expression for the signal in (1) and to derive a differential equation that includes the time varying $\alpha(t)$ and $\omega(t)$. It turns out the resulting differential equation can be transformed to a Bessel's equation of order $1 / 2$, for which there is a closed form solution. The solution can be used to integrate the system between samples, leading to an explicit expression for the discrete time model. Because the discrete time model is nonlinear, the states are estimated using an extended Kalman filter. Using simulations, this new algorithm is compared to the approaches based modified Cartesian model and the nearly constant frequency model, and it shown that the new algorithm has a lower tracking error, particularly when following harmonic signals where the frequency is changing rapidly.

The structure of the paper is as follows. Section II derives a differential equation that is satisfied by the harmonic signal when both amplitude and frequency are changing and shows how it can be reduced to the solution of a Bessel's equation of order $1 / 2$. Section III uses the solution to the differential equation to derive a discrete time model, which forms the basis of an extended Kalman filter. Section IV shows the results of applying the algorithm to simulated data and compares the performance with the outputs from extended Kalman filters based on the MCM and NCF models. Section V concludes the paper and outlines possible areas for developing the algorithm.

\section{DifFERENTIAL EQUATION}

Dropping the dependence on $t$ in order to simplify the notation and differentiating (1) gives

$$
\begin{aligned}
\frac{\mathrm{d} s}{\mathrm{~d} t} & =\frac{\mathrm{d} \alpha}{\mathrm{d} t} \cos \phi-\alpha \omega \sin \phi \\
\frac{\mathrm{d}^{2} s}{\mathrm{~d} t^{2}} & =\left(\frac{\mathrm{d}^{2} \alpha}{\mathrm{d} t^{2}}-\alpha \omega^{2}\right) \cos \phi-\left(2 \frac{\mathrm{d} \alpha}{\mathrm{d} t} \omega+\alpha \frac{\mathrm{d} \omega}{\mathrm{d} t}\right) .
\end{aligned}
$$

From (1) and (15)

$$
\begin{aligned}
\cos \phi & =\frac{1}{\alpha} s \\
\sin \phi & =\frac{1}{\alpha \omega} \frac{\mathrm{d} \alpha}{\mathrm{d} t}-\frac{1}{\alpha \omega} \frac{\mathrm{d} s}{\mathrm{~d} t} \\
& =\frac{1}{\alpha^{2} \omega} \frac{\mathrm{d} \alpha}{\mathrm{d} t} s-\frac{1}{\alpha \omega} \frac{\mathrm{d} s}{\mathrm{~d} t} .
\end{aligned}
$$

Substituting these expressions into (16) leads to

$$
\begin{aligned}
& s^{\prime \prime}+\left(-2 \frac{\alpha^{\prime}}{\alpha}-\frac{\omega^{\prime}}{\omega}\right) s^{\prime} \\
& \quad+\left(\omega^{2}+2\left(\frac{\alpha^{\prime}}{\alpha}\right)^{2}+\frac{\alpha^{\prime}}{\alpha} \frac{\omega^{\prime}}{\omega}-\frac{\alpha^{\prime \prime}}{\alpha}\right) s=0 .
\end{aligned}
$$



form

The differential equation in (20) can be expressed in the

$$
s^{\prime \prime}+g_{1}(t) s^{\prime}+g_{0}(t) s=0
$$

where

$$
\begin{aligned}
& g_{1}(t)=-2 \frac{\alpha^{\prime}}{\alpha}-\frac{\omega^{\prime}}{\omega} \\
& g_{0}(t)=\omega^{2}+2\left(\frac{\alpha^{\prime}}{\alpha}\right)^{2}+\frac{\alpha^{\prime}}{\alpha} \frac{\omega^{\prime}}{\omega}-\frac{\alpha^{\prime \prime}}{\alpha} .
\end{aligned}
$$

Using the substitution

$$
s(t)=u(t) \exp \left(-\frac{1}{2} \int g_{1}(t) \mathrm{d} t\right)
$$

the differential equation in $(21)$ can be reduced to

$$
u^{\prime \prime}+g(t) u=0
$$

where

$$
g(t)=g_{0}(t)-\frac{1}{4} g_{1}(t)^{2}-\frac{1}{2} g_{1}^{\prime}(t)
$$

From (22) and (23)

$$
\begin{aligned}
g_{1}(t)^{2} & =4\left(\frac{\alpha^{\prime}}{\alpha}\right)+4 \frac{\alpha^{\prime}}{\alpha} \frac{\omega^{\prime}}{\omega}+\left(\frac{\omega^{\prime}}{\omega}\right)^{2} \\
g_{1}^{\prime}(t) & =-2\left(\frac{\alpha \alpha^{\prime \prime}-\left(\alpha^{\prime}\right)^{2}}{\alpha^{2}}\right)-\left(\frac{\omega \omega^{\prime \prime}-\left(\omega^{\prime}\right)^{2}}{\omega^{2}}\right) \\
& =-2 \frac{\alpha^{\prime \prime}}{\alpha}+2\left(\frac{\alpha^{\prime}}{\alpha}\right)^{2}-\frac{\omega^{\prime \prime}}{\omega}+\left(\frac{\omega^{\prime}}{\omega}\right)^{2}
\end{aligned}
$$

so that

$$
\begin{aligned}
g(t) & =\omega^{2}+2\left(\frac{\alpha^{\prime}}{\alpha}\right)^{2}+\frac{\alpha^{\prime}}{\alpha} \frac{\omega^{\prime}}{\omega}-\frac{\alpha^{\prime \prime}}{\alpha}-\left(\frac{\alpha^{\prime}}{\alpha}\right)^{2}-\frac{\alpha^{\prime}}{\alpha} \frac{\omega^{\prime}}{\omega} \\
& -\frac{1}{4}\left(\frac{\omega^{\prime}}{\omega}\right)^{2}+\frac{\alpha^{\prime \prime}}{\alpha}-\left(\frac{\alpha^{\prime}}{\alpha}\right)^{2}+\frac{1}{2} \frac{\omega^{\prime \prime}}{\omega}-\frac{1}{2}\left(\frac{\omega^{\prime}}{\omega}\right)^{2} \\
& =\omega^{2}-\frac{1}{4}\left(\frac{\omega^{\prime}}{\omega}\right)^{2}+\frac{1}{2}\left[\frac{\omega^{\prime \prime}}{\omega}-\left(\frac{\omega^{\prime}}{\omega}\right)^{2}\right] \\
& =\omega^{2}-\frac{1}{4}\left(\frac{\omega^{\prime}}{\omega}\right)^{2}+\frac{1}{2} \frac{\mathrm{d}}{\mathrm{d} t}\left(\frac{\omega^{\prime}}{\omega}\right) \\
& =\omega^{2}-\frac{1}{4}\left[(\ln \omega)^{\prime}\right]^{2}+\frac{1}{2}(\ln \omega)^{\prime \prime}
\end{aligned}
$$

Assume that changes in $(\ln \omega)^{\prime}$ occur at each sampling time, so that between samples,

$$
(\ln \omega)^{\prime}=p
$$

where $p$ is a constant, so that

$$
(\ln \omega)^{\prime \prime}=0
$$

and

$$
\ln \omega=p t+q
$$

where $q$ is also constant. This means that

$$
g(t)=\mathrm{e}^{2 q} \mathrm{e}^{2 p t}-\frac{1}{4} p^{2}
$$

and from $(25)$

$$
\frac{\mathrm{d}^{2} u}{\mathrm{~d} t^{2}}+\left(\mathrm{e}^{2 q} \mathrm{e}^{2 p t}-\frac{1}{4} p^{2}\right) u=0 .
$$

Note that if $p=0$, then this reduces to

$$
\frac{\mathrm{d}^{2} u}{\mathrm{~d} t^{2}}+\mathrm{e}^{2 q} u=0
$$

or

$$
\frac{\mathrm{d}^{2} u}{\mathrm{~d} t^{2}}+\omega^{2} u=0
$$

which corresponds to a scaled version of the differential equation in (1) that describes the evolution of $s(t)$ when the frequency of the sinusoid is constant.

If $p^{2}>0$, then (38) can be rearranged as

$$
\frac{1}{p^{2}} \frac{\mathrm{d}^{2} u}{\mathrm{~d} t^{2}}+\left(\frac{1}{p^{2}} \mathrm{e}^{2 q} \mathrm{e}^{2 p t}-\frac{1}{4}\right) u=0 .
$$

Define

$$
\tau=\frac{1}{p} \mathrm{e}^{q} \mathrm{e}^{p t}
$$

so that

$$
\frac{\mathrm{d} \tau}{\mathrm{d} t}=p \tau
$$

and

$$
\begin{aligned}
\frac{\mathrm{d} u}{\mathrm{~d} t} & =\frac{\mathrm{d} \tau}{\mathrm{d} t} \frac{\mathrm{d} u}{\mathrm{~d} \tau}=p \tau \frac{\mathrm{d} u}{\mathrm{~d} \tau} \\
\frac{\mathrm{d}^{2} u}{\mathrm{~d} t^{2}} & =\frac{\mathrm{d} \tau}{\mathrm{d} t} \frac{\mathrm{d}}{\mathrm{d} \tau}\left(\frac{\mathrm{d} u}{\mathrm{~d} t}\right) \\
& =\frac{\mathrm{d} \tau}{\mathrm{d} t} \frac{\mathrm{d}}{\mathrm{d} \tau}\left(p \tau \frac{\mathrm{d} u}{\mathrm{~d} \tau}\right) \\
& =p^{2} \tau^{2} \frac{\mathrm{d}^{2} u}{\mathrm{~d} \tau^{2}}+p^{2} \tau \frac{\mathrm{d} u}{\mathrm{~d} \tau}
\end{aligned}
$$

Using this expression in (41) gives

$$
\tau^{2} \frac{\mathrm{d}^{2} u}{\mathrm{~d} \tau^{2}}+\tau \frac{\mathrm{d} u}{\mathrm{~d} \tau}+\left(\tau^{2}-\frac{1}{4}\right) u=0 .
$$

This is Bessel's equation of order $1 / 2$, which has a solution

$$
u(\tau)=C_{1} \sqrt{\frac{2}{\pi \tau}} \sin \tau+C_{2} \sqrt{\frac{2}{\pi \tau}} \cos \tau
$$

where $C_{1}$ and $C_{2}$ are constants. Substituting for $\tau$ from (42)

$$
\begin{aligned}
u(t)= & C_{1} \sqrt{\frac{2}{\pi}} \sqrt{p} \mathrm{e}^{-q / 2} \mathrm{e}^{-p t / 2} \sin \left(\frac{1}{p} \mathrm{e}^{p t+q}\right) \\
& +C_{2} \sqrt{\frac{2}{\pi}} \sqrt{p} \mathrm{e}^{-q / 2} \mathrm{e}^{-p t / 2} \cos \left(\frac{1}{p} \mathrm{e}^{p t+q} \cdot\right) .
\end{aligned}
$$


is

Using the expression for $g_{1}(t)$, the integrating factor in (24)

$$
\begin{aligned}
\exp \left[-\frac{1}{2} \int g_{1}(t) \mathrm{d} t\right] & =\exp \left[\frac{1}{2} \int\left(2 \frac{\alpha^{\prime}}{\alpha}+\frac{\omega^{\prime}}{\omega}\right) \mathrm{d} t\right] \\
& =\exp \left[\int(\ln \alpha)^{\prime} \mathrm{d} t+\frac{1}{2} \int(\ln \omega) \mathrm{d} t\right] \\
& =\exp \left[\ln \alpha+\frac{1}{2} \ln \omega\right] \\
& =\alpha \sqrt{\omega} \\
& =\alpha \mathrm{e}^{q / 2} \mathrm{e}^{p t / 2}
\end{aligned}
$$

Combining (50) and (55) in (24) gives

$$
\begin{array}{r}
s(t)=C_{1} \alpha(t) \sqrt{\frac{2}{\pi}} \sqrt{p} \sin \left(\frac{1}{p} \mathrm{e}^{p t+q}\right) \\
+C_{2} \alpha(t) \sqrt{\frac{2}{\pi}} \sqrt{p} \cos \left(\frac{1}{p} \mathrm{e}^{p t+q}\right)
\end{array}
$$

so that

$$
\begin{array}{r}
s^{\prime}(t)=C_{1} \sqrt{\frac{2}{\pi}} \sqrt{p}\left[\alpha^{\prime}(t) \sin \left(\frac{1}{p} \mathrm{e}^{p t+q}\right)\right. \\
\left.+\alpha(t) \mathrm{e}^{q} \mathrm{e}^{p t} \cos \left(\frac{1}{p} \mathrm{e}^{p t+q}\right)\right] \\
+C_{2} \sqrt{\frac{2}{\pi} \sqrt{p}}\left[\alpha^{\prime}(t) \cos \left(\frac{1}{p} \mathrm{e}^{p t+q}\right)\right. \\
\left.-\alpha(t) \mathrm{e}^{q} \mathrm{e}^{p t} \sin \left(\frac{1}{p} \mathrm{e}^{p t+q}\right)\right] .
\end{array}
$$

\section{Discrete Time State Space Model}

Define $x(t) \in \mathbb{R}^{5}$ as

$$
x^{\mathrm{T}}(t)=\left[\begin{array}{lllll}
s(t) & s^{\prime}(t) & (\ln \alpha(t))^{\prime} & (\ln \omega(t)) & (\ln \omega(t))^{\prime}
\end{array}\right]
$$

then the discrete time update of the state from $t=k T$ to $t=(k+1) T$ can be written in the form

$$
x[(k+1) T]=f(x[k T])+B w[k T]
$$

where $B^{\mathrm{T}}=\left[\begin{array}{lllll}0 & 0 & 1 & 0 & 1\end{array}\right]$ and $w[k T] \in \mathbb{R}^{2}$ is the state noise that affects $(\ln \alpha)^{\prime}$ and $(\ln \omega)^{\prime}$ at $t=k T$, which is taken to be zero-mean, Gaussian, white noise, $w(k T) \sim(0, Q)$, with $Q \in \mathbb{R}^{2 \times 2}$ being the covariance matrix of the noise.
Given $s(k T)$ and $s^{\prime}(k T)$, then (56) and (57) can be rearranged to provide expressions for $C_{1}$ and $C_{2}$

$$
\begin{aligned}
& C_{1}=\sqrt{\frac{\pi}{2}} \frac{1}{\sqrt{p}}\left\{\left[-\frac{A^{\prime}(0)}{A(0)^{2}} \mathrm{e}^{-q} \cos \left(\frac{1}{p} \mathrm{e}^{q}\right)\right.\right. \\
& \left.+\frac{1}{A(0)} \sin \left(\frac{1}{p} \mathrm{e}^{q}\right)\right] s(k T) \\
& \left.+\left[\frac{1}{A(0)} \mathrm{e}^{-q} \cos \left(\frac{1}{p} \mathrm{e}^{q}\right)\right] s^{\prime}(k T)\right\} \\
& C_{2}=\sqrt{\frac{\pi}{2}} \frac{1}{\sqrt{p}}\left\{\left[\frac{A^{\prime}(0)}{A(0)^{2}} \mathrm{e}^{-q} \sin \left(\frac{1}{p} \mathrm{e}^{q}\right)\right.\right. \\
& \left.+\frac{1}{A(0)} \cos \left(\frac{1}{p} \mathrm{e}^{q}\right)\right] s(k T) \\
& \left.-\left[\frac{1}{A(0)} \mathrm{e}^{-q} \sin \left(\frac{1}{p} \mathrm{e}^{q}\right)\right] s^{\prime}(k T)\right\} \text {. }
\end{aligned}
$$

Substituting these expressions back into (56) and (57) and assuming that $(\ln \omega)^{\prime}=p$ is constant over the interval, so that $\ln \omega=p(t-k T)+q$, where $\ln \omega(k T)=q$, leads to

$$
\begin{aligned}
& f_{1}(x)= x_{1} \mathrm{e}^{x_{3} T}\left\{\cos \left[\mathrm{e}^{x_{4}}\left(\frac{\mathrm{e}^{x_{5} T}-1}{x_{5}}\right)\right]\right. \\
&\left.-x_{3} \mathrm{e}^{-x_{4}} \sin \left[\mathrm{e}^{x_{4}}\left(\frac{\mathrm{e}^{x_{5} T}-1}{x_{5}}\right)\right]\right\} \\
&+ x_{2} \mathrm{e}^{x_{3} T}\left\{\mathrm{e}^{-x_{4}} \sin \left[\mathrm{e}^{x_{4}}\left(\frac{\mathrm{e}^{x_{5} T}-1}{x_{5}}\right)\right]\right\} \\
& f_{2}(x)= x_{1} \mathrm{e}^{x_{3} T}\left\{x_{3}\left(1-\mathrm{e}^{x_{5} T}\right) \cos \left[\mathrm{e}^{x_{4}}\left(\frac{\mathrm{e}^{x_{5} T}-1}{x_{5}}\right)\right]\right. \\
&+\left.-\left(x_{3}^{2} \mathrm{e}^{-x_{4}}+\mathrm{e}^{x_{3} T} \mathrm{e}^{x_{5} T}\right) \sin \left[\mathrm{e}^{x_{4}}\left(\frac{\mathrm{e}^{x_{5} T}-1}{x_{5}}\right)\right]\right\} \\
& f_{3} \mathrm{e}^{-x_{4}} \sin \left[\mathrm{e}^{x_{4}}\left(\frac{\mathrm{e}^{x_{5} T}-1}{x_{5}}\right)\right] \\
& f_{4}(x)=\left.x_{4}+\mathrm{e}^{x_{5} T} \cos \left[\mathrm{e}^{x_{4}}\left(\frac{\mathrm{e}^{x_{5} T}-1}{x_{5}}\right)\right]\right\} \\
& f_{3}(x)= x_{5} .
\end{aligned}
$$

For the state vector in (58), the measurement equation becomes

$$
y(k T)=c x(k T)+n(k T)
$$

where $c=\left[\begin{array}{lllll}1 & 0 & 0 & 0 & 0\end{array}\right]$.

Remark. One advantage of using the logarithm of the frequency and the amplitude (and the derivatives of these terms) in the state vector is that it automatically ensures that the estimates $\omega(k T)$ and $\alpha(k T)$ are positive. This avoids problems with large changes in phase associated with the estimates of amplitude or frequency switching between positive and negative values. 
TABLE I

RMS ERROR FOR ESTIMATES OF AMPLITUDE AND FREQUENCY SHOWN IN FIGURES 1 AND 2.

\begin{tabular}{|c|c|c|}
\hline & Amplitude & Frequency \\
\hline MCM & $9.16 \times 10^{-2}$ & 1.24 \\
\hline NCF & $6.80 \times 10^{-2}$ & $9.71 \times 10^{-1}$ \\
\hline FDTM & $5.27 \times 10^{-2}$ & $5.50 \times 10^{-1}$ \\
\hline New Method & $5.11 \times 10^{-2}$ & $3.33 \times 10^{-1}$ \\
\hline
\end{tabular}

Given initial estimates, $\hat{x}_{0 \mid-1}$ and $P_{0 \mid-1}$, the states can be estimated using an extended Kalman filter [23]

$$
\begin{aligned}
K_{k} & =P_{k \mid k-1} c^{\mathrm{T}}\left(c P_{k \mid k-1} c^{\mathrm{T}}+R\right)^{-1} \\
\hat{x}_{k \mid k} & =\hat{x}_{k \mid k-1}+K_{k}\left[y(k T)-c \hat{x}_{k \mid k-1}\right] \\
P_{k \mid k} & =\left(I-K_{k} c\right) P_{k \mid k-1} \\
\hat{x}_{k+1 \mid k} & =f\left(\hat{x}_{k \mid k}\right) \\
P_{k+1 \mid k} & =A_{k} P_{k \mid k} A_{k}^{\mathrm{T}}+B Q B^{\mathrm{T}}
\end{aligned}
$$

where $A_{k}$ denotes the Jacobian whose $i, j$ th element is

$$
\left[A_{k}\right]_{i, j}=\left.\frac{\partial f_{i}}{\partial x_{j}}\right|_{x=\hat{x}_{k \mid k-1}} .
$$

Explicit expressions can be derived for the terms in the Jacobian using the equations for $f(x)$ in (62) to (66). The EKF is implemented using a square root algorithm based on the QR factorisation [23].

\section{Simulation Results}

The proposed algorithm was applied to a noisy sinusoidal signal whose amplitude and frequency were both changing randomly. The mean value of the amplitude was $50 \mathrm{mV}$, while the mean value of the frequency was $90 \mathrm{~Hz}$. The standard deviation of the measurement noise was $5 \mathrm{mV}$. The results from the algorithm were compared with the estimates obtained from the modified Cartesian model (MCM) [15], the nearly constant frequency (NCF) model [16] and a simplified version of the model presented in this paper (FDTM) [24]. The sampling rate is $10 \mathrm{kHz}$. Figure 1 shows the estimates of the amplitude over a period of $1 \mathrm{~s}$, together with the error, while Figure 2 plots the estimated frequency. The results show that the new EKF algorithm outperforms both the MCM and the $\mathrm{NCF}$ algorithms, particularly for the estimate of the frequency, and it is also better than the simplified algorithm (FDTM). This is confirmed by the results in Table I, which gives the root mean square error (RMSE) for the estimates from each of the methods. It was found that the new algorithm was highly effective at tracking the signal when the frequency was changing rapidly. This is illustrated in Figure 3, which shows the the frequency estimate of the different algorithms when the frequency is reduced from $100 \mathrm{~Hz}$ to $85 \mathrm{~Hz}$ over a period of $0.5 \mathrm{~s}$.
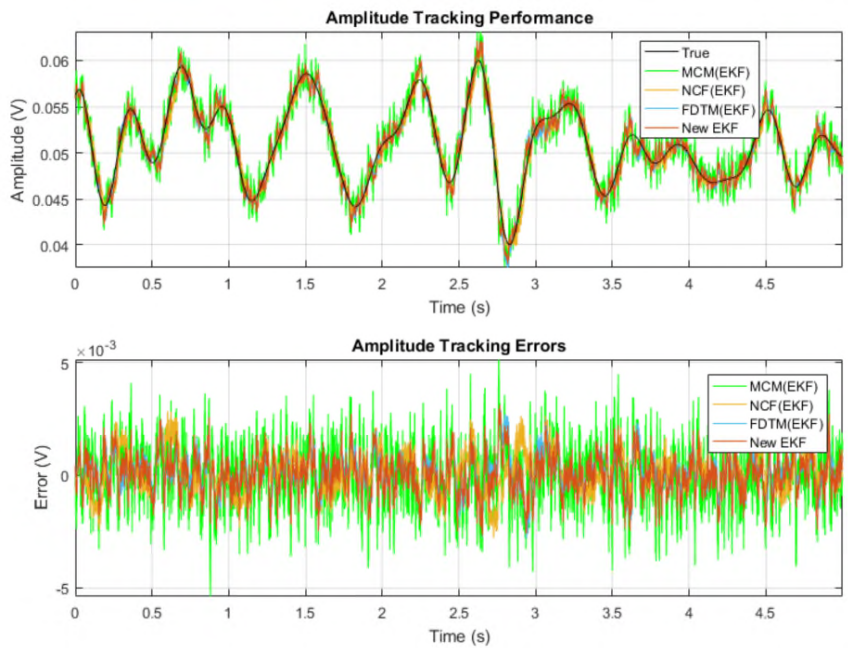

Fig. 1. Upper plot. Estimates of amplitude for different methods along with true value (black). Lower plot. Estimation errors.
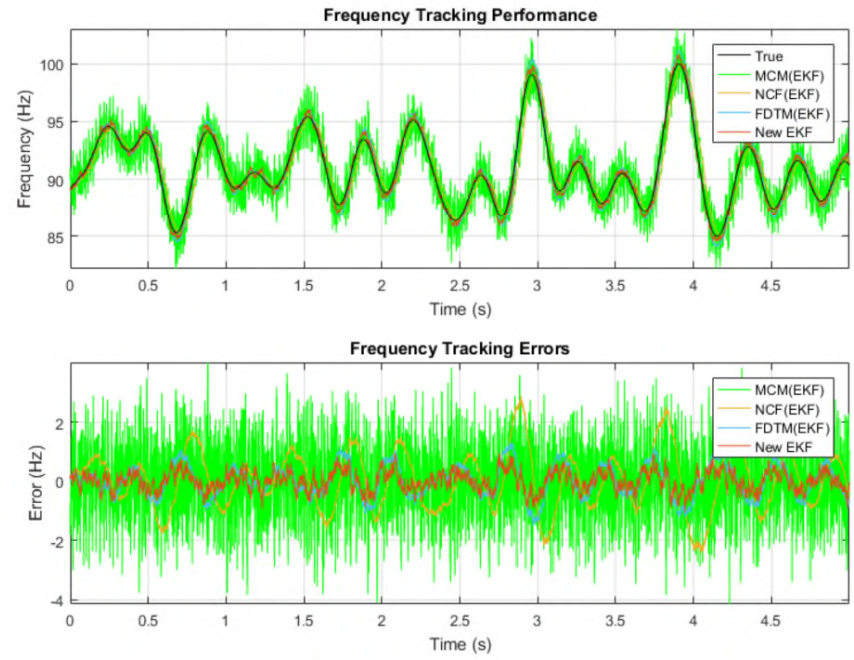

Fig. 2. Upper plot. Estimates of frequency for different methods along with true value (black). Lower plot. Estimation errors.

\section{CONCLUSION}

This paper has described a new model that forms the basis of an extend Kalman filter for extracting a harmonic signal from noisy measurements and estimating the the time varying amplitude and frequency of the signal. Unlike existing models, which are modifications of the model of a harmonic signal with constant amplitude and frequency, the model used here is based on a differential equation that is satisfied by the signal when both amplitude and frequency are changing. It is shown that this differential equation can be reduced to Bessel's equation of order $1 / 2$, which has a closed form solution. This solution is used to derive an explicit expression for a (nonlinear) discrete time state space model, which forms the basis of an extended Kalman filter. Simulations show 

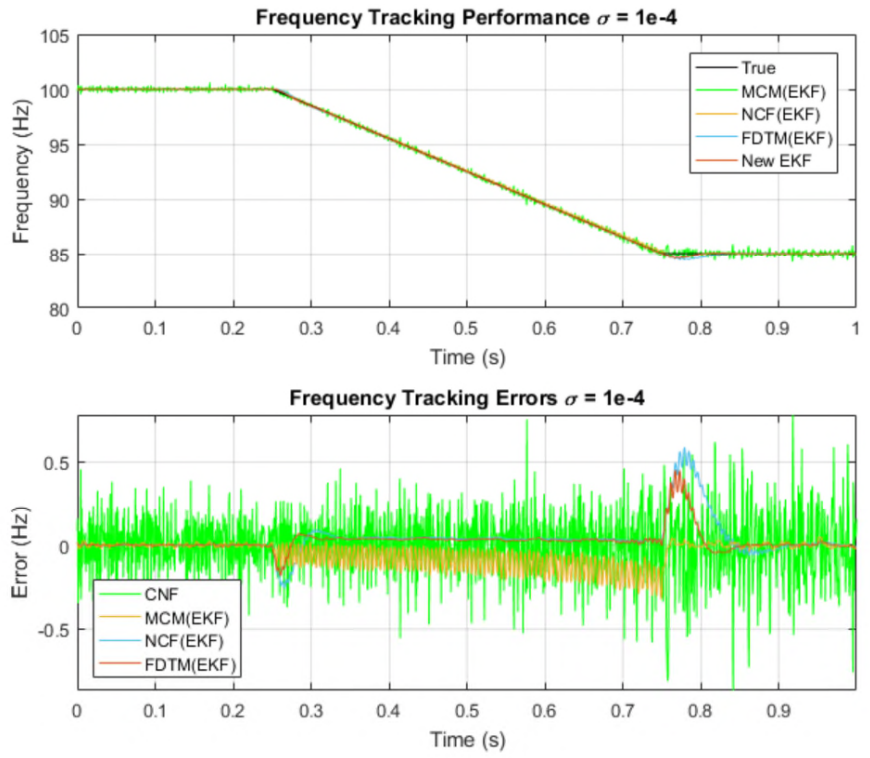

Fig. 3. Upper plot. Estimates of frequency for different methods along with true value (black) when following a ramp change. Lower plot. Estimation errors.

that this algorithms outperforms other approaches, particularly when tracking signals whose frequency is changing rapidly. An additional advantage of the algorithm is that by using the logarithm of the amplitude and frequency as states, this automatically ensures that the estimates of these variables are positive. However, the model is highly nonlinear, so it may be better to use an unscented Kalman filter, rather than an extended Kalman filter. This is currently being investigated.

\section{REFERENCES}

[1] M. Henry, "Self-validating digital Coriolis mass flow meter," Comput. Control Eng. J., vol. 11, no. 5, pp. 219-227, Oct. 2000

[2] R. Luke and J. Wouters, "Kalman filter based estimation of auditory steady state response parameters," IEEE Trans. Neural Syst. Rehabil. Eng., vol. 25, no. 3, pp. 196-204, Mar. 2017.

[3] S. Reza, M. Ciobotaru, and V. G. Agelidis, "Accurate estimation of single-phase grid voltage fundamental amplitude and frequency by using a frequency adaptive linear Kalman filter,' IEEE J. Emerg. Sel. Top. Power Electron., vol. 4, no. 4, pp. 1226-1235, Dec. 2016.

[4] X. Wang and E. E. Yaz, "Smart power grid synchronization with fault tolerant nonlinear estimation," IEEE Trans. Power Syst., vol. 31, no. 6, pp. 4806-4816, Nov. 2016.

[5] I. Sadinezhad and V. G. Agelidis, "Frequency adaptive least-squares Kalman technique for real-time voltage envelope and flicker estimation," IEEE Trans. Ind. Electron., vol. 59, no. 8, pp. 3330-3341, Aug. 2012.

[6] J.-L. Dion, C. Stephan, G. Chevallier, and H. Festjens, "Tracking and removing modulated sinusoidal components: A solution based on the kurtosis and the extended Kalman filter," Mech. Syst. Signal Process., vol. 38 , no. 2, pp. 428-439, Jul. 2013.

[7] S. Bittanti and S. M. Savaresi, "On the parametrization and design of an extended Kalman filter frequency tracker," IEEE Trans. Automat. Contr. vol. 45, no. 9, pp. 1718-1724, 2000.

[8] P. Regulski and V. Terzija, "Estimation of frequency and fundamental power components using an unscented Kalman filter," IEEE Trans. Instrum. Meas., vol. 61, no. 4, pp. 952-962, Apr. 2012.

[9] N.-K. C. Nair and J. J. Liu, "Estimation of power quality using an unscented Kalman filter," in Proc. TENCON 2007 - 2007 IEEE Region 10 Conference, Taipei, pp. 1-4, 2007
[10] F. Cupertino, E. Lavopa, P. Zanchetta, M. Sumner, and L. Salvatore, "Running DFT-based PLL algorithm for frequency, phase and amplitude tracking in aircraft electrical systems," IEEE Trans. Ind. Electron., vol. 58, no. 3, pp. 1027-1035, Mar. 2011.

[11] M. Chakraborty, "A new adaptive filter for estimating and tracking the delay and the amplitude of a sinusoid," IEEE Trans. Instrum. Meas., vol. 59, no. 11, pp. 3049-3057, Nov. 2010.

[12] G. Yin, L. Guo, and X. Li, "An Amplitude Adaptive Notch Filter for Grid Signal Processing," IEEE Trans. Power Electron., vol. 28, no. 6, pp. 2638-2641, Jun. 2013.

[13] D.L. Snyder, The State-Variable Approach to Continuous Estimation with Applications to Analog Communications Theory, MIT Press:Boston, MA, 1969.

[14] S. Gazor, H. Hajimolahoseini, H. Soltanian-Zadeh, and R. Amirfattahi, "Instantaneous fundamental frequency estimation of non-stationary periodic signals using non-linear recursive filters," IET Signal Process., vol. 9, no. 2, pp. 143-153, Apr. 2015.

[15] H. Hajimolahoseini, M. R. Taban, and H. Soltanian-Zadeh, "Extended Kalman filter frequency tracker for nonstationary harmonic signals," Measurement, vol. 45, no. 1, pp. 126-132, Jan. 2012.

[16] W. Ng, C. Ji, W. K. Ma, and H. C. So, "A study on particle filters for single-tone frequency tracking," IEEE Trans. Aerosp. Electron. Syst., vol. 45, no. 3, pp. 1111-1125, Jul. 2009.

[17] B. F. La Scala and R. B. Bitmead, "Design of an extended Kalman filter frequency tracker,", IEEE Trans. Automat. Contr, vol. 44, no. 3, pp. 739-742., March 1996.

[18] D. V. Bhaskar Rao and S. Y. Kung, "Adaptive notch filtering for the retrieval of sinusoids in noise," IEEE Trans. Acoustics, Speech, Signal Processing, vol. 32, pp. 791-802, Aug. 1984.

[19] A. Nehorai, "A minimal parameter adaptive notch filter with constrained poles and zeros," IEEE Trans. Acoustics, Speech, Signal Processing, vol. 33, no. 4, pp. 983-996, Aug. 1985.

[20] P. Handel and A. Nehorai, "Tracking analysis of an adaptive notch filter with constrained poles and zeros," IEEE Trans. Signal Processing, vol 42, pp. 281-291, Feb. 1994.

[21] S. Bittanti, M. Campi and S. M. Savaresi, "Unbiased estimation of a sinusoid in noise via adapted notch filters," Automatica, vol. 33, no. 2. pp. 209-215, 1997.

[22] B. F. La Scala, R. B. Bitmead and M. R. James, "Conditions for stability of the extended Kalman filter and their application to the frequency tracking problem," Math. Contr., Signals Syst., vol. 8, no. 1, pp. 1$27,1996$.

[23] T. Kailath, A. H. Sayed and B. Hassibi, Linear Estimation, Upper Saddle River, NJ:Prentice Hall, 2000.

[24] M. Li, Signal Processing for Next Generation Coriolis Flow Metering, D.Phil. thesis, Univerity of Oxford, Oxford, United Kingdom, UK, 2018. 